\title{
Thymosin Beta4 Regulates Cardiac Valve Formation Via Endothelial-Mesenchymal Transformation in Zebrafish Embryos
}

\author{
Sun-Hye Shin ${ }^{1,2}$, Sangkyu Lee ${ }^{1}$, Jong-Sup Bae ${ }^{1}$, Jun-Goo Jee ${ }^{1}$, Hee-Jae Cha ${ }^{3}$, and You Mie Lee ${ }^{1,2, \star}$
}

Thymosin beta4 (TB4) has multiple functions in cellular response in processes as diverse as embryonic organ development and the pathogeneses of disease, especially those associated with cardiac coronary vessels. However, the specific roles played by TB4 during heart valve development in vertebrates are largely unknown. Here, we identified a novel function of TB4 in endothelialmesenchymal transformation (EMT) in cardiac valve endocardial cushions in zebrafish. The expressions of thymosin family members in developing zebrafish embryos were determined by whole mount in situ hybridization. Of the thymosin family members only zTB4 was expressed in the developing heart region. Cardiac valve development at $48 \mathrm{~h}$ post fertilization was defected in zebrafish TB4 (zTB4) morpholino-injected embryos (morphants). In zTB4 morphants, abnormal linear heart tube development was observed. The expressions of bone morphogenetic protein (BMP) 4, notch1b, and hyaluronic acid synthase (HAS) 2 genes were also markedly reduced in atrio-ventricular canal (AVC). Endocardial cells in the AVC region were stained with anti-Zn5 antibody reactive against Dm-grasp (an EMT marker) to observe EMT in developing cardiac valves in zTB4 morphants. EMT marker expression in valve endothelial cells was confirmed after transfection with TB4 siRNA in the presence of transforming growth factor $\beta$ (TGF $\beta$ ) by RT-PCR and immunofluorescent assay. Zn5-positive endocardial AVC cells were not observed in zTB4 morphants, and knockdown of TB4 suppressed TGF- $\beta$-induced EMT in ovine valve endothelial cells. Taken together, our results demonstrate that TB4 plays a pivotal role in cardiac valve formation by increasing EMT.

\footnotetext{
${ }^{1}$ Research Institute of Pharmaceutical Sciences, College of Pharmacy, ${ }^{2}$ School of Life Sciences and Biotechnology, College of Natural Sciences, Kyungpook National University, Daegu 702-701, Korea, ${ }^{3}$ Department of Parasitology and Genetics, Kosin University College of Medicine, Busan 602-703, Korea

*Correspondence: lym@knu.ac.kr

Received 9 January, 2014; revised 20 February, 2014; accepted 28 February, 2014; published online 8 April, 2014
}

Keywords: endothelial-mesenchymal transformation, heart valve formation, thymosin beta4, zebrafish

\section{INTRODUCTION}

Thymosin beta 4 (TB4) is a water-soluble 43-amino acid polypeptide that does not possess signal sequences for secretion or nuclear localization signaling (NLS). TB4 has numerous functions, the most prominent of which involves the sequestration of G-actin monomers and the actin-cytoskeletal organization necessary for cell motility, organogenesis, and other cellular events (Huff et al., 2001). TB4 promotes skin and corneal wound healing by enhancing the endothelial cell migration, proliferation, differentiation, and the survival of a variety of cells (Malinda et al., 1997). It has been reported that TB4 can activate the survival kinase Akt and play a potent role in the protection of cardiac muscle from cell death after ischemic damage, as occurs in the setting of myocardial infarction (MI) (BockMarquette et al., 2004). TB4 can also promote angiogenesis in ischemic areas (Bock-Marquette et al., 2009; Smart et al., 2006), contribute to heart wound healing (Malinda et al., 1997; 1999) and promote cancer metastasis (Sribenja et al., 2013) and inflammatory responses (Huff et al., 2001; Sosne et al., 2002). Furthermore, TB4 has been reported to prime epicardiumderived progenitors to differentiate into cardiomyocytes (Smart et al., 2011) or coronary vessels (Bock-Marquette et al., 2009). Thus, it appears that TB4 has pleiotropic effects in various cellular responses, which included the promotion of cardiac repair (Bock-Marquette et al., 2004; Goldstein et al., 2012; Thatcher et al., 2012), cancer growth, and metastasis (Ji et al., 2013; Sribenja et al., 2013). TB4 has been reported to be present at elevated levels in the developing blood vessels and heart endocardial cushions of early mouse embryos post-implantation (Gomez-Marquez, 1996), which suggests it plays a key role in the formation of the cardiovascular system. TB4 is upregulated by tenascin- $C$ signaling, which is likely involved in valve calcification ( $\mathrm{Li}$ et al., 2002). However, the precise role played and the mechanism of TB4 in valve formation has not been investigated.

The developmental processes involved in heart formation have widely studied in experimental animal models, and it is known that cardiac valve formation is initially started by myocardial inductive signals in the non-chamber region. The human heart tube is composed of an outer layer of myocardium and an inner lining of endocardial cells, separated by an extensive extracellular matrix (ECM), which is referred to as cardiac jelly. After rightward looping of the heart, the cardiac jelly overlying 
the future atrioventricular canal (AVC) and outflow tract (OT) expands into cardiac cushions (Derynck and Zhang, 2003). Endothelial mesenchymal transformation (EMT) is an essential event characterized by delamination and invasion of cardiac jelly by endocardial cells in cardiac cushions (Markwald et al., 1977). Therefore, we tried to determine whether TB4 is involved in EMT during cardiac valve formation. In this study, we used a zebrafish model because this model offers several distinct advantages as a genetic and developmental model system for studies of cardiovascular defects. Furthermore, this model allows investigation of genes required for cardiovascular development because the zebrafish has a transparent heart during the early stage of heart development (Bakkers, 2011). During zebrafish heart development, heart looping starts at $36 \mathrm{~h}$ postfertilization (hpf) and functional valves are almost completely formed by $48 \mathrm{hpf}$, although valve development is completed at around $55 \mathrm{hpf}$ (Stainier et al., 2002). In this study, we found that the only member of the zTB family expressed in lateral plate mesoderm (LMP) during the early stage and in the heart region in the late stage was zTB4. Furthermore, knock-down of zTB4 with antisense morpholino oligomers induced linear heart tube formation and prevented AVC formation and the expressions of AVC marker genes. However, zTB4 gene rescued heart defects and the AVC marker gene loss observed in zTB4 morphants. Knock-down of zTB4 also attenuated EMT marker gene expressions in zebrafish AVC and in sheep endothelial cells induced by TGF $\beta 1$. Taken together, we suggest that TB4 plays an essential role in the regulation of EMT in zebrafish AVC and facilitates heart valve development.

\section{MATERIALS AND METHODS}

\section{Fish stock and maintenance}

Zebrafish strains were maintained as previously described (Westerfield, 1993). Animal experiments were performed according to the guidelines for the care and use of laboratory animals issued by the institutional ethical animal care committee of Kyungpook National University (Korea). The AB strain was used as a wild-type control. We maintained transgenic lines expressing enhanced green fluorescent protein (EGFP) under the control of a cmlc2 promoter, Tg(cmlc2:EGFP) (Huang et al., 2003), and flk-1 promoter, Tg(flk-1:EGFP) ${ }^{\mathrm{s} 843}$ (Jin et al., 2005). Embryos were dechorionated with pronase at each developmental stage, and treated with $0.003 \% 1$-phenyl2-thio-urea (PTU) (Sigma) to inhibit pigment formation.

\section{Morpholino antisense oligonucleotide (MO) injection and the rescue experiment}

zTB4 morpholino was synthesized by Gene-Tool, LLC (USA); its sequence was 5'-AGGTCTGAAAAAGGAAATGACAGGA-3'. $M O$ was resuspended in diethylpyrocarbonate (DEPC)-treated water and then injected into one-or two-cell stage embryos collected by mating transgenic zebrafish using a micro-injector (M3301R, WPI, USA)

Whole mount in situ hybridization

Digoxigenin-labeled antisense RNA probes specific for zTB1, zTB2, zTB4, zTB10, cmlc2, notch1b, bmp4, and hyaluronan synthase 2 (HAS2) were prepared and used for whole mount in situ hybridization, as previously described (Kim et al., 2002). Stained embryos were mounted in glycerol and examined using a Normarski differential interference contrast lens (Axioskop, Zeiss, Germany).
Table 1. PCR primer sequences

\begin{tabular}{|c|c|}
\hline Gene name & Primers sequences \\
\hline TB4 & $\begin{array}{l}\text { F : 5'-CTCCTTCTCCTGACCAGCTT-3', } \\
\text { R : 5'-CTGCTTCTCCTGTTCGATGG-3' }\end{array}$ \\
\hline$\alpha-S M A$ & $\begin{array}{l}\text { F : 5'-TCTTTGAAGCCAACGACCTG-3', } \\
\text { R : 5'-ATCTGCAGGCTGACGTTGAG-3' }\end{array}$ \\
\hline CD31 & $\begin{array}{l}\mathrm{F}: 5^{\prime} \text {-CCT GACATCTCCACCAATG-3', } \\
\mathrm{R}: 5^{\prime} \text {-TAAGATCCGTCAAGGGAGCC-3' }\end{array}$ \\
\hline$\beta$-actin & $\begin{array}{l}\mathrm{F}: 5^{\prime} \text {-GATCCACATCTGCTGGAA-3', } \\
\mathrm{R}: \text { 5'-GACTACCTCATGAAGATC-3' }^{\prime} \text { ' }\end{array}$ \\
\hline
\end{tabular}

Whole mount fluorescence immunostaining

Whole mount zebrafish embryos were immunostained with a mouse monoclonal antibody against Zn5 (1:500; Hybridoma Bank, USA) and Alexa Fluor 594-conjugated anti-mouse IgG antibody (1:1000, Molecular Probes, USA) as a secondary antibody. Images of the immunostained hearts were obtained with a Zeiss LSM5 Pascal confocal microscope.

\section{Cell culture}

Sheep aortic valve endothelial cells (AVECs) were kindly provided Dr. Joyce Bischoff's Lab in Children's Hospital, Boston (Harvard Medical School) (Paranya et al., 2001) and cultured on gelatin-coated tissue culture plates at $37^{\circ} \mathrm{C}$ in a humidified atmosphere incubator containing $5 \% \mathrm{CO}_{2}$ in EBM (Hyclone, USA) supplemented with $20 \%$ fetal bovine serum (FBS, Hyclone) and antibiotics. Ovine AVECs were used at passages 710.

\section{Small interfering (si) RNA-mediated gene knockdown in} endothelial cells

A pool of 4 distinct siRNAs directed against TB4 was synthesized by Qiagen Genosolution siRNA (USA). Subconfluent sheep valve endothelial cells (passage 6-10) were transfected with TB4 siRNA using a microporator (Invitrogen, USA).

Semi-quantitative reverse transcriptase polymerase chain reaction (RT-PCR)

Total RNA was extracted using TRIzol reagent (Invitrogen) and cDNA synthesis from total RNA was performed using the first strand cDNA synthesis kit (Promega, USA). cDNA was used as a template for PCR-reactions, which were conducted using specific primers (Table 1).

\section{Western blot analysis}

Equal amounts of proteins were separated by SDS-polyacrylamide gel electrophoresis (PAGE) and transferred to nitrocellulose membranes (Whatman, England). After blocking membranes with $5 \%$ non-fat milk in Tris buffered saline (TBS) containing $0.1 \%$ Tween 20 for $1 \mathrm{~h}$ at RT, membranes were incubated with the appropriate primary antibody at $4^{\circ} \mathrm{C}$ overnight, and then with horseradish peroxidase-conjugated mouse or rabbit IgG antibody at RT for $1 \mathrm{~h}$. Blots were developed using West Pico chemiluminescent substrate (PIERCE, USA).

Immunofluorescence (IF) assay

Ovine aortic endothelial cells on coverslips were fixed with $10 \%$ formaldehyde solution for $10 \mathrm{~min}$ at RT. Cells were then per 
A

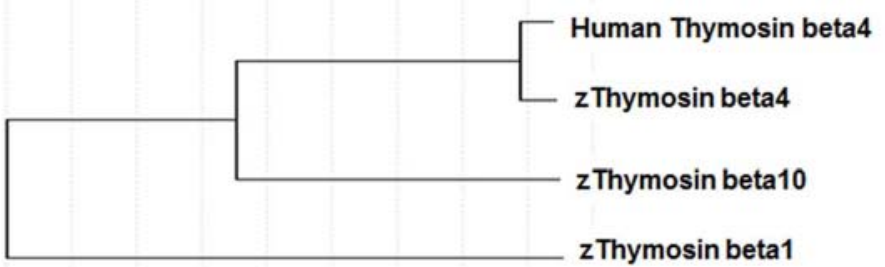

B

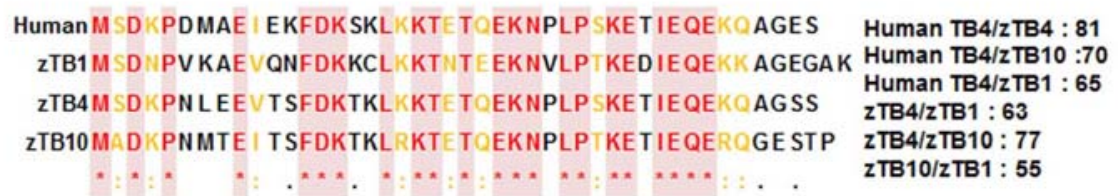

C

Human M SDKPD M AE I EKFDK SKLKK TET QEKNPLP SKETI EQEKQAG ES Human/Mouse: 100 Mouse M SDKP D MAE I EKFDK SKLKK TETQEKN PLP SKETI EQEKQAG ES Human/Zebrafish: 81 Zebrafish M S DKPN LEEVTSFDK TKLKK TET QEKNPLP SKETIEQEKQAG S S Mouse/Zebrafish: 81

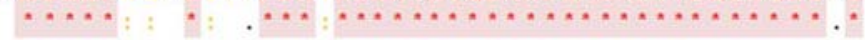

\section{D}
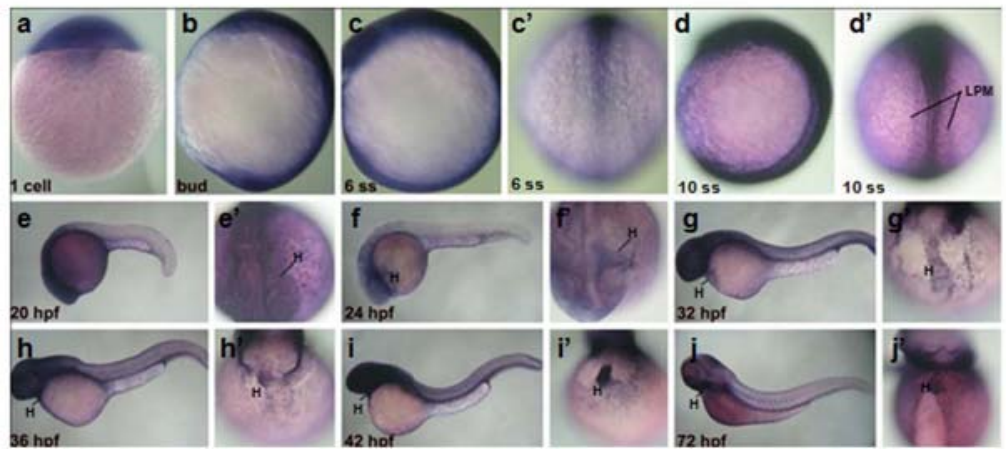

Fig. 1. Homology and molecular phylogenetic analysis of thymosin family in vertebrates. (A) Molecular phylogenetic tree of human TB4 and the zebrafish thymosin family. (B) Multiple sequence alignment of human TB4 and zebrafish TB family members. (C) Multiple sequence alignment of the TB proteins of different species. The boxed sequences indicate the Gactin binding sites of TB family members. (D) Whole mount in situ hybridization of zTB4 was performed in zebrafish embryos from a zygote to 10 ss (a-d') during zebrafish embryogenesis, as described in "Materials and Methods". Lateral (b, c, d) and dorsal $\left(c^{\prime}, d^{\prime}\right)$ views are shown. zTB4 expression was detected in the heart region from $20 \mathrm{hpf}$ to 72 hpf $\left(e^{\prime}, f^{\prime}, g ; h^{\prime}, i^{\prime}\right.$ and $j^{\prime}$ : ventral view; e, $f, g, h, i$ and $j$ : lateral view) (E) Expression of zTB4 at 10 ss (d') was detected throughout lateral plate mesoderm (LPM). meabilized with $0.5 \%$ TritonX-100 in PBS for $10 \mathrm{~min}$, and blocked with $1 \%$ BSA in PBS. After blocking, anti-CD31 and anti- $\alpha$-smooth muscle actin ( $\alpha$-SMA) antibodies in PBS containing $1 \% \mathrm{BSA}$ were added at $4^{\circ} \mathrm{C}$ overnight. The cells were then washed with PBS-T and incubated with anti-mouse FITCconjugated and anti-goat Texas red-conjugated antibodies for 1 $h$. The cells on coverslips were then washed with PBS-T and mounted with DAPI mounting solution onto slides. Fluorescent images were obtained using a Zeiss fluorescence microscope (LSM5, Pascal, Germany).

\section{RESULTS}

Bioinformatic analysis of the thymosin family

To start a comparative analysis on sequence homologies of thymosin family members in zebrafish and man, we used the Genebee database and compared human TB4 with zebrafish thymosin family amino acid sequences. Phylogenetic analysis showed that human TB4 (hTB4; NM021109) protein clustered with zebrafish TB1 (zTB1; DQ989583), zebrafish zTB4 (zTB4; DQ119892), and zebrafish TB10 (zTB10; BC115156) (Fig. 1A). Multiple sequence alignment (MSA) analysis revealed high similarity between zTB4 and hTB4. More specifically, the per- centage similarity between zTB4 and hZTB4 was $81 \%$, and the amino acid sequences of G-actin sequestering sites in ZTB family and hTB4 were identical (Figs. $1 B$ and $1 C$ ). MSA revealed $81-100 \%$ identity at the protein level between zTB4 and its orthologs in man (NM021109) and in mouse (NM021278) (Fig. 1C).

Thymosin family was expressed from the early stage of zebrafish development

Expression of thymosin family in early zebrafish embryos was analyzed with zTB family anti-sense probes by whole mount in situ hybridization. Weak expressions of zTB1 and zTB10 were observed during the whole developmental stages (Supplementary Figs. 1A and 1C), but zTB2 and zTB4 were detected. The expression of zTB2 was localized in the developing brain, that is, in hindbrain (HB), telencephalon (TC), and tegmentum (TG) (Supplementary Fig. 1B). zTB2 was expressed in the neural plate between the zygote and the 10 somite stage (ss), that is, at $20 \mathrm{hpf}$ (lateral view, Supplementary Fig. 1C, e). At $24 \mathrm{hpf}$, zTB2 was expressed in HB, TC, and TG, and its neuronal expression persisted at $42 \mathrm{hpf}$, but was weak at $72 \mathrm{hpf}$ (Supplementary Fig. 2B, i, j). In contrast, zTB4 was expressed in the head and anterior regions of embryos, and was specifically 

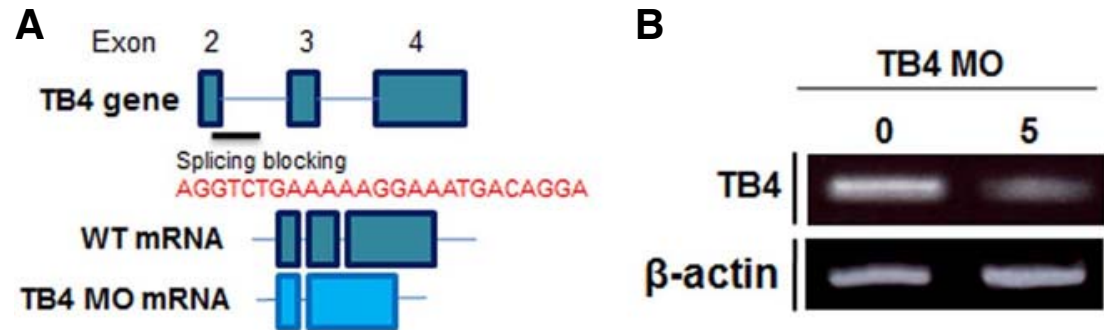

C
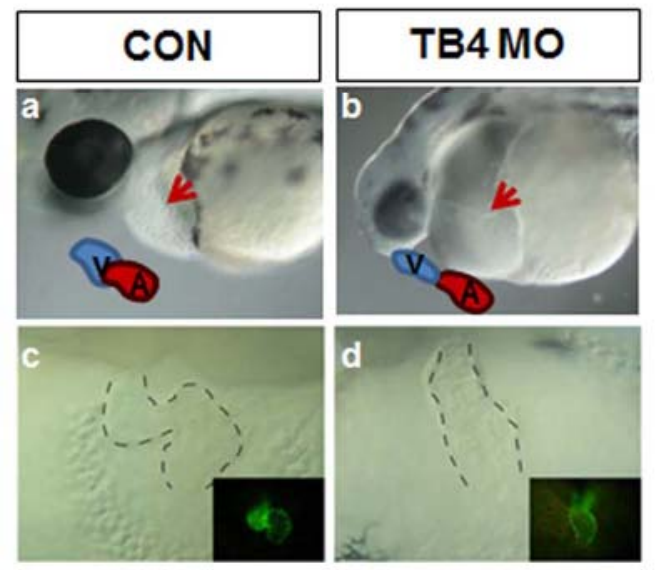

Fig. 2. Site and sequences of the antisense morpholino oligomers used for blocking zTB4 splicing and the phenotype of zTB4 morphants. (A) The upper diagram shows zTB4 gene exons 2-4. ZTB4 MO was designed to block splicing of exon 2 (ATG TCTGAC) and intron 2 (CTGCA AAC) of zTB4 pre-mRNA (resultant gene diagrams are indicated below $\mathrm{MO}$ sequences). (B) After $\mathrm{MO}(5 \mathrm{ng})$ injection into one cell embryo, the expression levels of zTB4 and $\beta$ actin mRNA were determined by RT-PCR in total RNA. (C) Morphologies of control embryos $(a, c)$ and zTB4 MO-injected embryos (b, d) at $48 \mathrm{hpf}$ were observed under a stereomicroscope. a, b; lateral view. Drawings inside pictures indicate the ventricle $(\mathrm{V})$ and atrium $(\mathrm{A})$ and the arrow indicates the heart tube. c, d; frontal view. Dotted lines show the heart tube. $\mathrm{Tg}$ (cmlc2:EGFP) embryos showing heart tube morphology in the dark images located inside the bright field pictures in (c, d). expressed in lateral plate mesoderm (LPM) (Fig. 1D, d'), which develops to produce many organs including the heart $(H)$ (Bakkers et al., 2009), suggesting the possibility that zTB4 may be involved in heart organogenesis. Flat images of $10 \mathrm{ss}$ embryos revealed the obvious expression of zTB4 in LPM (Fig. $1 \mathrm{E})$ and zTB4 expression in myocardium throughout hearts (Fig. 1D). Therefore, we speculated that of the zTB family members, only zTB4 is involved in heart development.

\section{Knockdown of thymosin beta4 induced developmental heart defects}

Because zTB4 was found to be expressed in heart, we sought to determine its function during embryonic heart development. Accordingly, we designed splicing inhibitory morpholinos that target the splice donor site of exon E2 to knockdown zTB4 (Fig. 2A). As shown in Fig. 2B, zTB4 morpholino was effective at disrupting the splicing of zTB4 pre-mRNA, and resulted in remarkable reductions in mature mRNA levels. Based on the severity of phenotypic changes observed at $48 \mathrm{hpf}$, we categorized the morphants into three types (Supplementary Fig. 2). Type I embryos with heart edema were used in further experiments. We confirmed that the first obvious phenotype was pericardial edema at $48 \mathrm{hpf}$, indicating a cardiac defect (Fig. 2C). zTB4 morphants had a linearized morphology (Fig. 2C, b, d) in contrast to control embryos, which showed a s-shaped looping heart morphology at this time in the valve region (Fig. $2 \mathrm{C}, \mathrm{a}, \mathrm{c}$ ). These observations indicate that zTB4 might regulate looping of the heart during development.

Thymosin beta 4 regulated the expressions of genes required for heart valve formation in the atrio-ventricular canal

It has been reported that heart valve development is tightly regulated by signaling between endocardial and underlying myocardial cell layers in the atrio-ventricular canal (AVC) (Johnson et al., 2003). Glycosaminoglycan hyaluronic acid (HA) exists as a hydrated gel that expands the extracellular space, regulates ligand availability, and interacts with numerous ECM components including proteoglycan (versican), a major constituent of cardiac jelly in the AVC (Armstrong and Bischoff, 2004). To investigate the underlying basis of chamber maturation and normal cardiac tube looping, we examined the expression of the cardiac specific marker, cardiac myosin light chain 2 (cm/c2) (Yelon et al., 1999). In zTB4 morphants, cmlc2 expressing cells formed a linear heart tube (Fig. 3e), whereas control embryos formed a S-loop heart tube (Fig. 3a). To determine whether defective cell differentiation in the AVC was responsible for abnormal heart looping in zTB4 morphants, we analyzed the expressions of myocardial and endocardial markers in AVCs. The expression of bmp4 in AVC myocardial cells (Armstrong and Bischoff, 2004; Milan et al., 2006) was broad and ectopic (Fig. 3f), and the expressions of HAS2 and notch $1 b$ in AVC endocardial cells were markedly lower in morphants (Figs. $3 \mathrm{~g}$ and $3 \mathrm{~h}$ ) than in controls (Figs. 3b, 3c, and 3d). However, when we rescued the zTB4 gene in zTB4 morphants, heart edema and cardiac marker gene expressions recovered to control embryo levels, suggesting that zTB4 might be essential for AVC development during cardiac valve formation (Figs. 3i-3I). These results indicate that zTB4 plays an essential role in maturation, heart tube looping, and in the formation of heart valves.

\section{Knockdown of thymosin beta4 suppressed EMT in AVC} endocardial cells

To confirm the role of zTB4 during heart valve formation, we examined the differentiation of endocardial AVC cells in zTB4 morphants. Anti-Zn5 antibody recognizes Dm-grasp, a cellsurface adhesion molecule of the immunoglobulin superfamily 


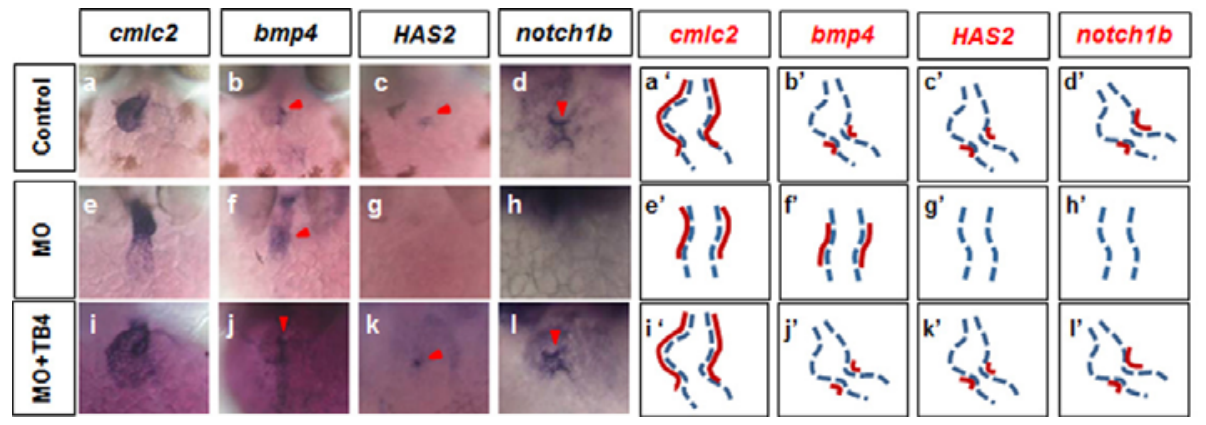

$\left(a^{\prime}-l^{\prime}\right)$ are schematic diagrams of the heart tube region (dashed line) and the expression patterns of each gene (red line).
Fig. 3. Expression of endocardial and EMT marker genes in AVCs were disturbed in zTB4 morpholinoinjected zebrafish embryos. (A) Whole mount in situ hybridization of embryos at $48 \mathrm{hpf}$ was performed using riboprobes for cmlc2, bmp4, HAS2, and notch1b. Staining for cmlc2 (a, e, i), bmp4 (b, f, j), HAS2 (c, g, k), and notch1b (d, h, l) was observed in controls (a-d), zTB4 morholino (MO) (e-h), and MO plus zTB4 mRNA (i-l) injected embryos.
A

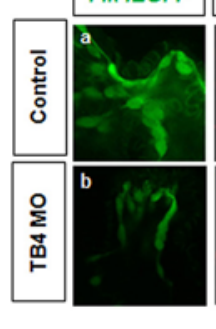

C

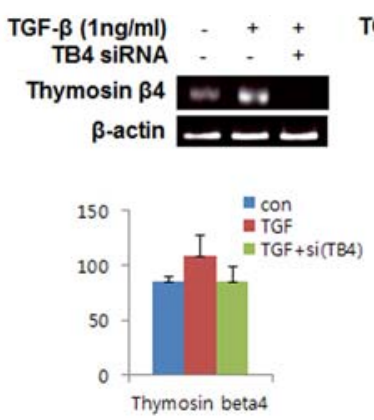

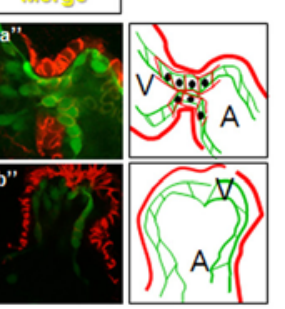
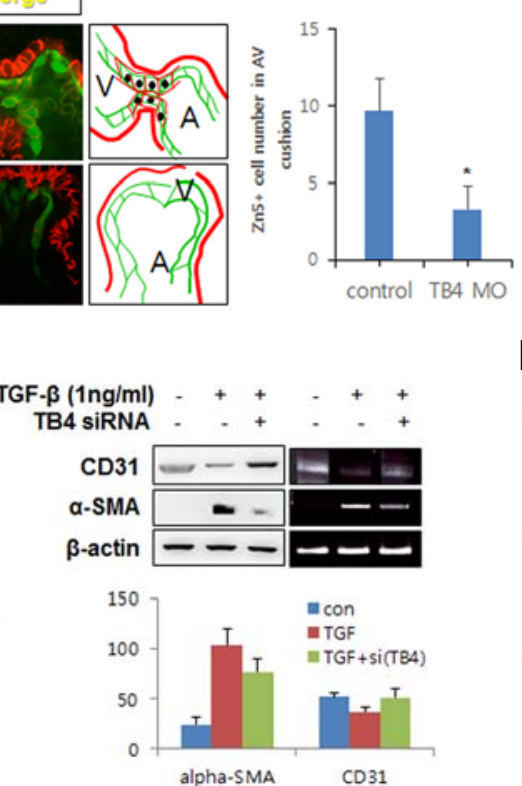

B

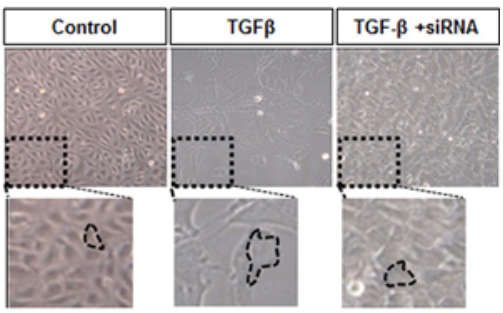

D

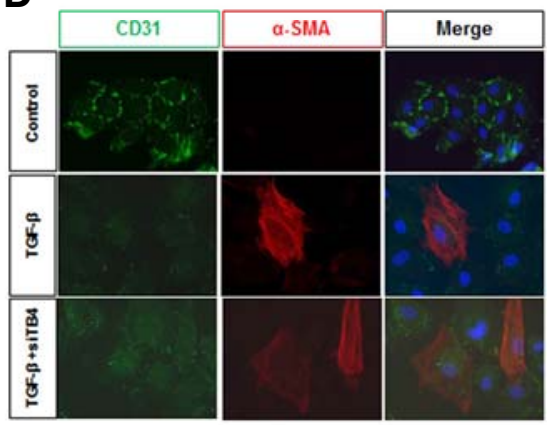

Fig. 4. Thymosin $\beta 4$ regulated EMT during zebrafish heart valve formation. (A) Endocardial cells expressing GFP in control Tg(flk: EGFP) embryos were immunostained with anti-Zn5 antibody at $48 \mathrm{hpf}$ in control (arrowhead, $\mathrm{a}^{\prime}$ ) and zTB4 morphants (arrowhead, $\mathrm{b}^{\prime}$ ) and observed under a confocal microscope (X800). Green (endocardial cells) and red fluorescent (Zn5: EMT marker) cells are shown in the schematic diagram shown in right boxes ( $\mathrm{V}$; ventricle, A; atrium). Numbers of $\mathrm{Zn5^{+ }}$ cells were counted and plotted (right panel) $(\mathrm{n}=3)$. (B) Aortic valve endothelial cells were treated with TGF $\beta 1(1 \mathrm{ng} / \mathrm{ml})$ for 8 days in EBM basal media in the presence or absence of TB4 siRNA. Cell morphologies were observed; results are shown in the upper pictures and in magnified images in the lower panels. (C) Semiquantitative RT-PCR for thymo$\sin \beta 4$ and $\beta$-actin mRNA was performed using the same samples in (B) (left panel) $(n=3)$. Cell lysates obtained from the same samples were analyzed for the expressions of CD31 and $\alpha$-SMA protein and mRNA by Western blotting (left) or semiquantitative RT-PCR (right) (right panel). $\beta$-Actin was used as an internal loading control. The quantitative expression of each gene are plotted $(n=3)$. (D) Aortic valve endothelial cells were grown for 8 days in the absence or presence of TGF- $\beta$ or TB4 siRNA in EBM basal media. Cells were immunostained with anti-CD31 and anti- $\alpha$-SMA antibodies. Green (CD31) and red fluorescent $(\alpha-S M A)$ cells were observed under a confocal microscope. Scale bar $=10 \mu \mathrm{m}$.

in zebrafish AVC cushions (Beis et al., 2005), and enables the visualization of myocardial and endocardial cells undergoing EMT in AVCs. In the AVC region of control Tg(flk:EGFP) hearts, differentiating endocardial cells were positive for Zn5 (Fig. 4A, $a^{\prime}$, arrows) and GFP, indicating the presence of EMT. However, Zn5-positive differentiating endocardial AVC cells were markedly diminished in the AVC region of zTB4 morphants (Fig. 4A, b', arrows), suggesting that EMT for heart valve formation hardly occurs in the absence of zTB4. Furthermore, the number of $\mathrm{Zn5}^{+}$cells in the AVC region was significantly lower in TB4 morphant embryos (Fig. 4A, right graph). These results suggest that $Z$ TB4 participates in the EMT required for heart valve development by upregulating Zn5 in zebrafish embryos.

To confirm the role played by TB4 in the EMT of valve endothelial cells, we treated sheep aortic valve endothelial cells with TGF- $\beta 1(1 \mathrm{ng} / \mathrm{ml})$ for 8 days, because TGF- $\beta 1$ is known to in- 
duce EMT in these cells (Paranya et al., 2001). TGF- $\beta 1$-treated cells demonstrated slower growth kinetics and exhibited a spindle-shaped morphology instead of the cobble-stone morphology of non-treated control cells (Fig. 4B). Upregulation of TB4 mRNA expression was observed in TGF- $\beta 1$-treated cells, while knockdown of TB4 by small interfering (si) RNA clearly abolished TGF- $\beta 1$-induced TB4 gene expression (Fig. 4C). Furthermore, TB4 mRNA expression was upregulated in TGF- $\beta 1$ treated cells, and knockdown of TB4 by small interfering (si) RNA clearly abolished TGF- $\beta 1$-induced TB4 gene expression (Fig. $4 C$ ). In addition, TGF- $\beta 1$ induced $\alpha$-smooth muscle actin (SMA) expression but decreased CD31 expression at the mRNA and protein levels. However, knockdown of TB4 recovered CD31 but decreased $\alpha$-SMA in the presence of TGF- $\beta 1$, suggesting that TB4 is necessary for TGF- $\beta 1$-induced EMT (Fig 4C). Furthermore, immunofluorescent assays confirmed these findings (Fig. 4D). Taken together, our results demonstrate that TB4 is involved in the EMT required for cardiac valve cushion formation in zebrafish embryos.

\section{DISCUSSION}

Here, we identified for the first time that TB4 is a regulator of heart valve formation in zebrafish embryos. TB4 has multiple functions in cellular responses during embryonic organ development and pathogenic states, but the role of TB4 in heart valvular development has not been well identified. Valvular developmental mechanisms should be identified because adult heart valve diseases, such as, cardiac valve fibrosis can be treated by redirecting or amplifying disease-related EMT. In the present study, zTB4 was expressed in developing LPM and in the heart valve region. zTB4 morphants showed a linear heart tube and reduced expressions of valve marker genes, whereas recovery of the zTB4 gene in morphants restored abnormal phenotypes and gene expressions. The EMT marker protein, Dm-grasp was stained with Zn5 antibody to observe distinct cellular differentiation patterns in valve regions. However, Zn5 staining disappeared in AVC linear heart tubes in zTB4 morphants, indicating that ZTB4 is essential for the EMT required for cardiac valve formation.

EMT is a process that generates valve mesenchyme and this process is followed by endocardial cell proliferation, which elongate valve leaflets (Armstrong and Bischoff, 2004). TGF $\beta$ is a growth factor that induces EMT (Paranya et al., 2001), but this process is inhibited by VEGF in isolated valve endothelial cells (Yang et al., 2008). VEGF signaling has been importantly implicated during valve formation, and VEGFR2 acts to direct morphogenesis in AVC cushions (Lee et al., 2006), whereas VEGFR1 signaling does in the outflow tract (Stankunas et al., 2010). Furthermore, it has been shown that TB4 mediates the transcriptions of a number of genes, including VEGF and matrix metalloproteinases (MMPs), and therefore, increases endothelial cell migration and angiogenesis (Dube et al., 2012). TB4 also induces angiogenesis via Notch signaling in endothelial cells, but VEGF and Notch1 play opposing roles in the regulation of EMT in valve endothelium (Yang et al., 2008). NFATc1, a valve marker, functions to inhibit EMT (Yang et al., 2008) and increases the proliferation of endocardium and mesenchyme. Moreover, the activation of NFATc1 by VEGF (Johnson et al., 2003) explains the negative regulation of EMT by VEGF. Based on these findings, TB4 could regulate valve formation by inhibiting EMT via VEGF. However, TB4 induces TGF $\beta$ - 1 and laminin-5 2 chain in corneal epithelial cells (Sosne et al., 2004), and we found TB4 increased EMT marker genes, and that the ab- sence of TB4 clearly inhibited EMT in zebrafish AVC cushions and ovine valve endothelial cells (Fig. 4). In our previous study, VEGF was found to increase the expressions of a variety of genes responsible for EMT homeostasis by activating NFATc1 (Jang et al., 2010), which suggests that TB4 might regulate EMT homeostasis by increasing VEGF and/or through other pathways, such as, TGF $\beta 1$ signaling pathway. Because in the present study, knockdown of TB4 was found to reduce the expression of bmp4 (Fig. 3), we supposed that the BMP signaling pathway might contribute to the TB4-induced EMT required for valve formation. Actually, it has been reported that TB4 activation increases BMP2 and 4 and Smad protein levels in odontoblast cells (Lee et al., 2013).

TB4 has been suggested to have therapeutic potential for cardiac repair and regeneration because of its pleiotropic effects on various cellular responses, such as, wound healing, angiogenesis, and cardiomyocyte survival (Dube et al., 2012). In addition to its role in the regeneration of cardiomyocytes after myocardiac infarction, it might also be useful for cardiac valve regeneration in congenital heart disease or adult heart valve fibrosis by increasing EMT. In conclusion, our results demonstrate that TB4 participates in valve formation by promoting EMT in cardiac cushions. However, the detailed molecular and cellular mechanisms involved in the regulation of EMT in valve endocardial cells by TB4 remain to be determined.

Note: Supplementary information is available on the Molecules and Cells website (www.molcells.org).

\section{ACKNOWLEDGMENTS}

We thank the Korean Zebrafish Organogenesis Mutant Bank (ZOMB) in Kyungpook National University for providing the zebrafish lines and molecular markers used in this study, and Dr. Joyce Bischoff [(Children's Hospital, Boston (Harvard Medical School)] for generously providing sheep aortic valve endothelial cells (AVECs). This research was supported by a National Research Foundation (NRF) grant funded by the Korean government (Minstry of Sience, ICT and Future Planning) (NRF-2012R1A4A1028835).

\section{REFERENCES}

Armstrong, E.J., and Bischoff, J. (2004). Heart valve development: endothelial cell signaling and differentiation. Circ. Res. 95, 459470.

Bakkers, J. (2011). Zebrafish as a model to study cardiac development and human cardiac disease. Cardiovasc. Res. 91, 279288.

Bakkers, J., Verhoeven, M.C., and Abdelilah-Seyfried, S. (2009). Shaping the zebrafish heart: from left-right axis specification to epithelial tissue morphogenesis. Dev. Biol. 330, 213-220.

Beis, D., Bartman, T., Jin, S.W., Scott, I.C., D'mico, L.A., Ober, E.A., Verkade, H., Frantsve, J., Field, H.A., Wehman, A., et al. (2005). Genetic and cellular analyses of zebrafish atrioventricular cushion and valve development. Development 132, 4193-4204.

Bock-Marquette, I., Saxena, A., White, M.D., DiMaio, J.M., and Srivastava, D. (2004). Thymosin $\beta 4$ activates integrin-linked kinase and promotes cardiac cell migration, survival and cardiac repair. Nature 432, 466-472.

Bock-Marquette, I., Shrivastava, S., Pipes, G., Thatcher, J.E., Blystone, A., Shelton, J.M., Galindo, C.L., Melegh, B., Srivastava, D., and Olson, E.N. (2009). Thymosin $\beta 4$ mediated PKC activation is essential to initiate the embryonic coronary developmental program and epicardial progenitor cell activation in adult mice in vivo. J. Mol. Cell. Cardiol. 46, 728-738.

Derynck, R., and Zhang, Y.E. (2003). Smad-dependent and Smadindependent pathways in TGF-beta family signalling. Nature 425, 577-584. 
Dube, K.N., Bollini, S., Smart, N., and Riley, P.R. (2012). Thymosin beta4 protein therapy for cardiac repair. Curr. Pharm. Des. 18, 799-806.

Goldstein, A.L., Hannappel, E., Sosne, G., and Kleinman, H.K. (2012). Thymosin $\beta 4$ : a multi-functional regenerative peptide. Basic properties and clinical applications. Exp. Opin. Biol. Ther. 12, 37-51.

Gomez-Marquez, J., Franco del Amo, F., Carpintero, P., and Anadon, R. (1996). High levels of mouse thymosin $\beta 4$ mRNA in differentiating P19 embryonic cells and during evelopment of cardiovascular tissues. Biochim. Biophys. Acta 1306, 187-193.

Huang, C.J., Tu, C.T., Hsiao, C.D., Hsieh, F.J., and Tsai, H.J. (2003) Germ-line transmission of a myocardium-specific GFP transgene reveals critical regulatory elements in the cardiac myosin light chain 2 promoter of zebrafish. Dev. Dyn. 228, 30-40.

Huff, T., Müller, C.S., Otto, A.M., Netzker, R., and Hannappel, E. (2001). $\beta$-Thymosins, small acidic peptides with multiple functions. Int. J. Biochem. Cell Biol. 33, 205-220.

Jang, G.H., Park, I.S., Yang, J.H., Bischoff, J., and Lee, Y.M. (2010). Differential functions of genes regulated by VEGF-NFATc1 signaling pathway in the migration of pulmonary valve endothelial cells. FEBS Lett. 584, 141-146.

Ji, Y.I., Lee, B.Y., Kang, Y.J., Jo, J.O., Lee, S.H., Kim, H.Y., Kim, Y.O., Lee, C., Koh, S.B., Kim, A., et al. (2013). Expression patterns of Thymosin $\beta 4$ and cancer stem cell marker CD133 in ovarian cancers. Pathol. Oncol. Res. 19, 237-245.

Jin, S.W., Beis, D., Mitchell, T., Chen, J.N., and Stainier, D.Y.R (2005). Cellular and molecular analyses of vascular tube and lumen formation in zebrafish. Development 132, 5199-5209.

Johnson, E.N., Lee, Y.M., Sander, T.L., Rabkin, E., Schoen, F.J., Kaushal, S., and Bischoff, J. (2003). NFATc1 mediates vascular endothelial growth factor-induced proliferation of human pulmonary valve endothelial cells. J. Biol. Chem. 278, 1686-1692.

Kim, S.H., Shin, J., Park, H.C., Yeo, S.Y., Hong, S.K., Han, S., Rhee, M., Kim, C.H., Chitnis, A.B., and Huh, T.L. (2002). Specification of an anterior neuroectoderm patterning by Frizzled8a-mediated Wnt8b signalling during late gastrulation in zebrafish. Development 129, 4443-4455

Lee, Y.M., Cope, J.J., Ackermann, G.E., Goishi, K., Armstrong, E.J., Paw, B.H., and Bischoff, J. (2006). Vascular endothelial growth factor receptor signaling is required for cardiac valve formation in zebrafish. Dev. Dyn. 235, 29-37.

Lee, S.I., Kim, D.S., Lee, H.J., Cha, H.J., and Kim, E.C. (2013). The role of thymosin beta 4 on odontogenic differentiation in human dental pulp cells. PLoS One 8, e61960.

Li, Q., Jones, P., Lafferty, R., Safer, D., and Levy, R. (2002). Thymosin beta4 regulation, expression and function in aortic valve interstitial cells. J. Heart Valve Dis. 11, 726-735.

Malinda, K., Goldstein, A., and Kleinman, H. (1997). Thymosin beta 4 stimulates directional migration of human umbilical vein endothelial cells. FASEB J. 11, 474-481.

Malinda, K.M., Sidhu, G.S., Mani, H., Banaudha, K., Maheshwari, R.K., Goldstein, A.L., and Kleinman, H.K. (1999). Thymosin $\beta 4$ accelerates wound healing. J. Invest. Dermatol. 113, 364-368.
Markwald, R.R., Fitzharris, T.P., and Manasek, F.J. (1977). Structural development of endocardial cushions. Am. J. Anat. 148, 85-119.

Milan, D.J., Giokas, A.C., Serluca, F.C., Peterson, R.T., and MacRae, C.A. (2006). Notch1b and neuregulin are required for specification of central cardiac conduction tissue. Development 133, 1125-1132.

Paranya, G., Vineberg, S., Dvorin, E., Kaushal, S., Roth, S.J., Rabkin, E., Schoen, F.J., and Bischoff, J. (2001). Aortic valve endothelial cells undergo transforming growth factor-beta-mediated and non-transforming growth factor-beta-mediated transdifferentiation in vitro. Am. J. Pathol. 159, 1335-1343.

Smart, N., Risebro, C.A., Melville, A.A., Moses, K., Schwartz, R.J., Chien, K.R., and Riley, P.R. (2006). Thymosin beta4 induces adult epicardial progenitor mobilization and neovascularization. Nature 445, 177-182.

Smart, N., Bollini, S., Dubé, K.N., Vieira, J.M., Zhou, B., Davidson, S., Yellon, D., Riegler, J., Price, A.N., and Lythgoe, M.F. (2011) De novo cardiomyocytes from within the activated adult heart after injury. Nature 474, 640-644.

Sosne, G., Szliter, E.A., Barrett, R., Kernacki, K.A., Kleinman, H. and Hazlett, L.D. (2002). Thymosin beta 4 promotes corneal wound healing and decreases inflammation in vivo following alkali injury. Exp. Eye Res. 74, 293-299.

Sosne, G., Xu, L., Prach, L., Mrock, L.K., Kleinman, H.K., Letterio, J.J., Hazlett, L.D., and Kurpakus-Wheater, M. (2004). Thymosin beta 4 stimulates laminin-5 production independent of TGF-beta. Exp. Cell Res. 293, 175-183

Sribenja, S., Wongkham, S., Wongkham, C., Yao, Q., and Chen, C. (2013). Roles and mechanisms of $\beta$-thymosins in cell migration and cancer metastasis: an update. Cancer Invest. 31, 103-110.

Stainier, D.Y., Beis, D., Jungblut, B., and Bartman, T. (2002). Endocardial cushion formation in zebrafish. Cold Spring Harb. Symp. Quant. Biol. 67, 49-56.

Stankunas, K., Ma, G.K., Kunnert, F.J., Kuo, C.J., and Chang, C.P. (2010). VEGF signaling has distinct spatiotemporal roles during heart valve development. Dev. Biol. 347, 325-336.

Thatcher, J.E., Welch, T., Eberhart, R.C., Schelly, Z.A., and Michael DiMaio, J. (2012). Thymosin $\beta 4$ sustained release from poly (lactide-co-glycolide) microspheres: synthesis and implications for treatment of myocardial ischemia. Ann. N Y Acad. Sci. 1270 112-119.

Westerfield, M. (1993). The zebrafish book: a guide for the laboratory use of zebrafish (Brachydanio rerio), (University of Oregon Press, USA).

Yang, J.H., Wylie-Sears, J., and Bischoff, J. (2008). Opposing actions of Notch1 and VEGF in post-natal cardiac valve endothelial cells. Biochem. Biophys. Res. Commun. 374, 512-516.

Yelon, D., Horne, S.A., and Stainier, D.Y. (1999). Restricted expression of cardiac myosin genes reveals regulated aspects of heart tube assembly in zebrafish. Dev. Biol. 214, 23-37. 